(C) 2008 IEEE. Personal use of this material is permitted. Permission from IEEE must be obtained for all other uses, in any current or future media, including reprinting/republishing this material for advertising or promotional purposes, creating new collective works, for resale or redistribution to servers or lists, or reuse of any copyrighted component of this work in other works. 


\title{
CRUISE RESEARCH ACTIVITIES TOWARD UbiQUITOUS INTELLIGENT SENSING ENVIRONMENTS
}

\author{
Anelia Mitseva and Neeli R. Prasad, Aalborg University \\ Petia Todorova, FraUnHOFER FOKUS \\ RAMON AGUERO, UNIVERSITY OF CANTABRIA \\ ANA GARCIA ARMADA, UNIVERSITY CARLOS III OF MADRID \\ ChRISTOS PANAYIOTOU, UNIVERSITY OF CYPRUS \\ ANDREAS TIMM-GIEL, UNIVERSITY OF BREMEN \\ LEONARDO MACCARI, UNIVERSITY OF FLORENCE
}

\begin{abstract}
Wireless sensor networks are expected to be one of the key enabling technologies in the near future. Ubiquitous intelligent sensing environments have a promising potential to enhance the everyday life of citizens, bringing important social benefits for each person and for society as a whole. Substantial research has contributed to progress in this field. However, there are still gaps to be filled. This article presents an overview of the Creating Ubiquitous Intelligent Sensing Environments (CRUISE) project and identifies challenges in the research on WSNs. It focuses on outlining the consortium's vision and strategy for the evolution of research. Key issues for target sensor network applications and current research orientations in CRUISE are described. Future work within the project focus areas is identified.
\end{abstract}

\section{INTRODUCTION}

Working toward ubiquitous intelligent sensing environments, we are already witnessing the promising future of this technology with trial prototypes and early systems. Wireless sensor networks (WSNs) are under development in vehicles, smart houses, road and traffic safety applications, for emergency response, health monitoring, support for elder people, monitoring of farm animals [1], and other uses. The wide diversity of applications leads to different requirements and challenges, making multidisciplinary research efforts necessary. Nevertheless, the great potential for innovative user-centric applications [2] is a major driving force motivating researchers to work on a variety of challenging research topics. WSNs' unique feature is that they can capture the spa- tial and temporal dynamics of the environment or process they monitor.

Although many protocols and algorithms have been proposed for traditional wireless ad hoc networks, they are not well suited to the diverse architectural features and application requirements of sensor networks. The nature of WSNs adds extra requirements - the protocols and algorithms must cope with processing power, energy, remote configuration, and deployment constraints. Hence, self-assembly and continuous self-organization during the lifetime of the network in an efficient, reliable, secure, and scalable manner are crucial for the successful deployment and operation of such networks. Furthermore, cross-layer optimization plays an important role; on one hand for efficient coordination of data aggregation (sensing), and on the other hand in order to realize energy-efficient reliable communications with limited processing capabilities. In summary, sensor networks pose a number of new conceptual and optimization problems and many implementation challenges.

The Creating Ubiquitous Intelligent Sensing Environments (CRUISE) Network of Excellence, with 32 partners (http://www.ist-cruise. $\mathrm{eu} /$ ), started in 2006, and focuses on research in WSNs and the integration of researchers on this topic. Focal points of the joint research activities are some highly relevant areas: architecture and topology, protocols and data fusion, mobility and security, and transmission, as presented later. The novelty of the CRUISE approach is to deal simultaneously with applications and basic research issues so that applications open new research topics, and basic research solutions make new services possible.

Within CRUISE, challenging topics have been defined, and integrated work is under- 
way in order to provide common solutions toward the successful implementation of ubiquitous intelligent sensing environments. Some of the investigated research topics are classical problems of WSNs that have not been completely solved yet; others are quite new.

The article continues with key applications, the focus of the work, current research orientations, and open issues in the four identified integration areas; and concludes with an outlook for future work.

\section{TARGet Applications OF Wireless SENSOR NETWORKS}

In a wide range of applications such as environment and habitat monitoring, asset tracking, smart homes, and healthcare, WSNs will lead to more effectiveness, cost reduction, and increased quality of life. Applications like environmental and health monitoring, vehicle support, and event tracking are being investigated in CRUISE.

\section{ENVIRONMENTAL MONITORING}

WSNs are expected to find wide applicability in environment and habitat monitoring since they can easily be deployed in large areas, and capture the spatial and temporal state of the monitored environment. Of relevance here is event detection and localization, where an event can be the outbreak of a forest fire or release of a toxic substance in the drinking water reservoir [3]. A sensor field is deployed over a large area, and nodes measure various signals (e.g., temperature or pollutant concentration). The main problem is to use the measurements to decide the existence of an event and locate its source.

\section{LOGISTICS MANAGEMENT}

Atomization of goods, new value chains, just-intime production, globalization, and further developments in logistics require new technical solutions. WSNs and advanced communication will allow better surveillance of goods and even enable decentralized decision making at goods transport, as investigated in the framework of Collaborative Research Center 637 funded by the German Research Foundation. Sensors and radio frequency identification (RFID) tags can be placed in transport vehicles (Fig. 1), as well as on goods.

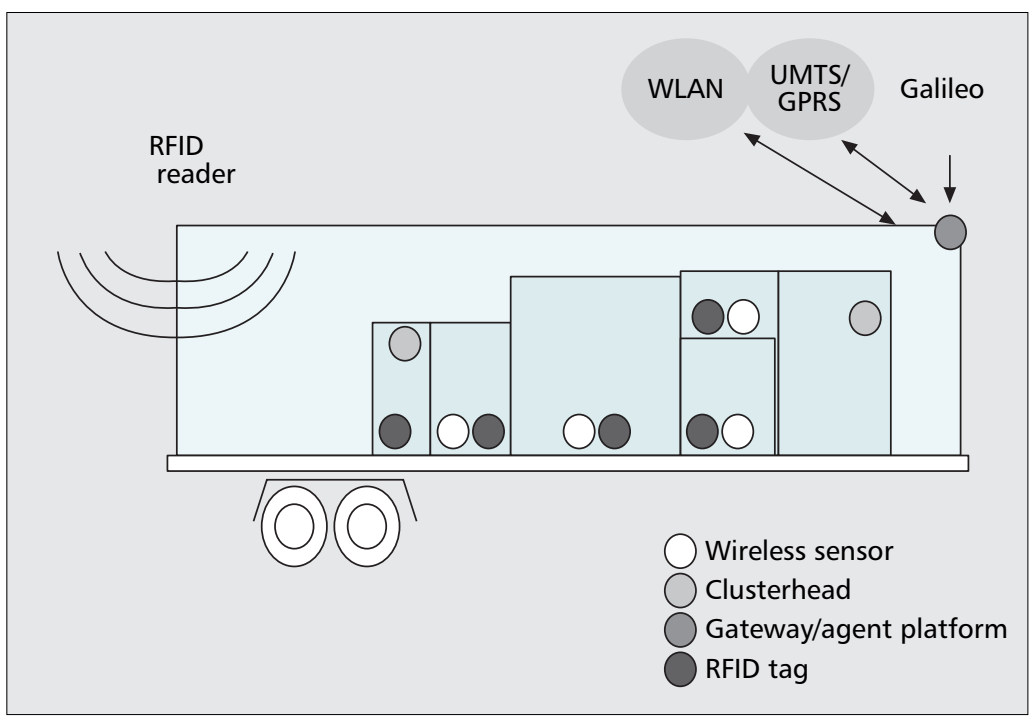

Figure 1. Logistic management: intelligent container.

\section{EMERGENCY MANAGEMENT}

In emergency management (e.g., firefighting and emergency aid after disasters), emergency forces cannot assume the presence of any communication infrastructure, but sensor information from different locations can improve their work. In a disaster scenario the same technology would enable medics to more effectively care for large numbers of casualties. An integration of WSNs with firefighters is demonstrated in [4]; the focus is on the use of WSNs to directly support the firefighter when entering a building on fire (Fig. 2).

\section{HEALTH MONITORING}

In medical care, outfitting care subjects with tiny wearable wireless sensors forming a body sensor network (BSN) would allow medical teams to monitor the status of their patients (at either the hospital or home). In this case the BSN transmits the current readings of vital signs (heart beat rate, body temperature, blood glucose, etc.) to a hospital database. Medical staff, emergency teams, and general practitioners can then access the data when needed (Fig. 3).

These applications are very diverse, and have different needs in terms of large-/small-scale deployment, interference-free/interference-prone environment, information velocity required, and passive nodes/actuators. This diversity motivates the research challenges introduced next.

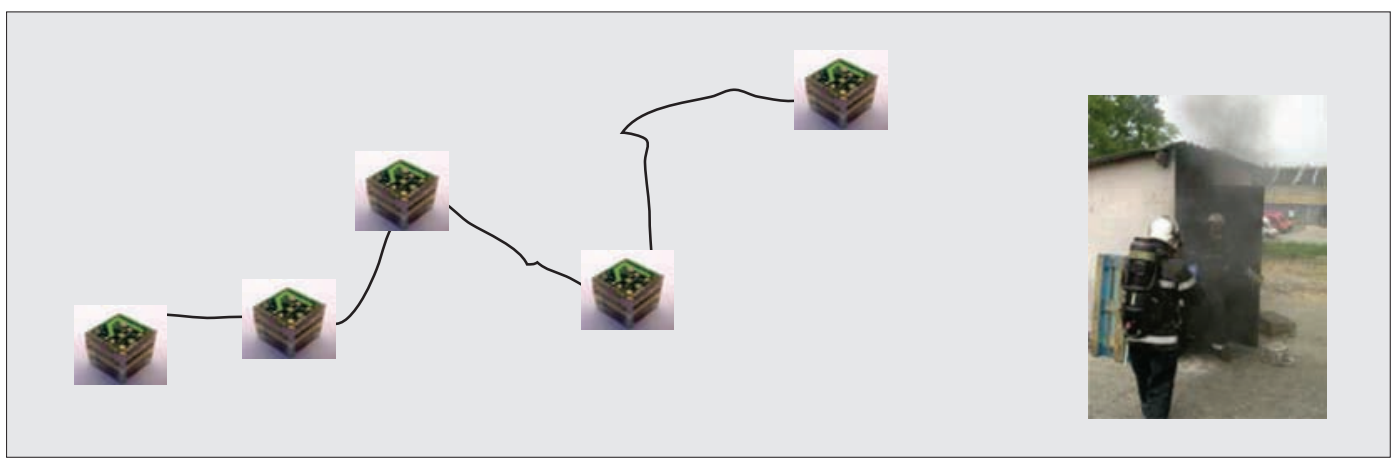

Figure 2. Firefighting scenario: virtual lifeline relaying voice messages and mission-critical data. 


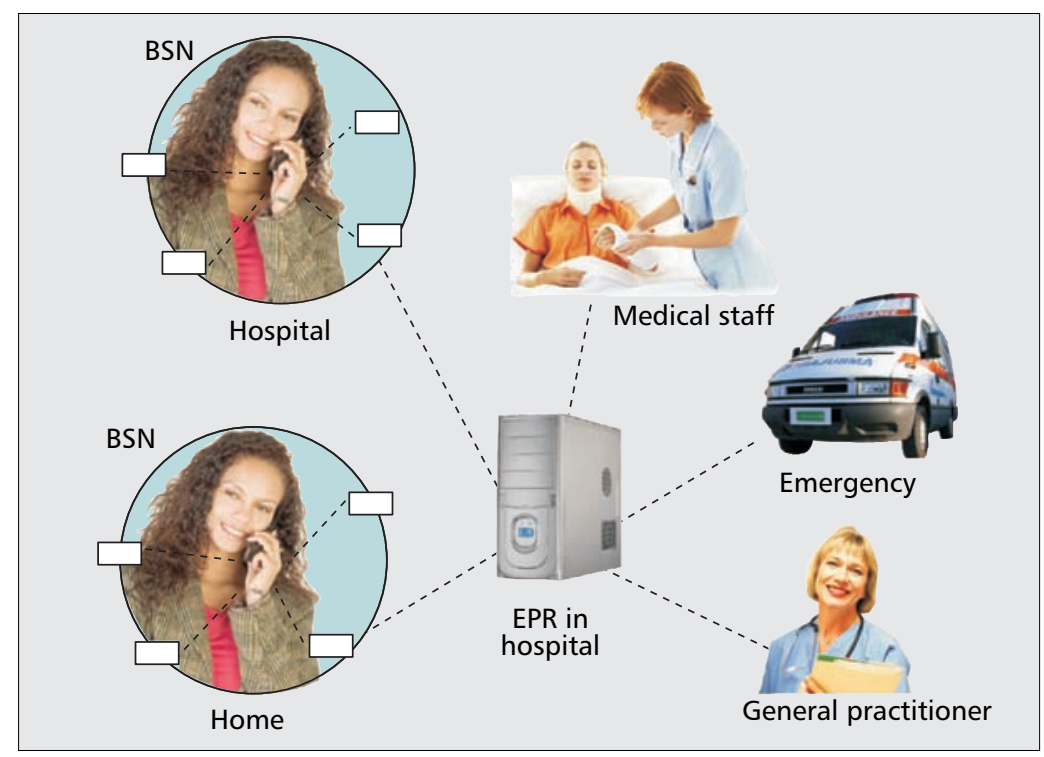

Figure 3. Health monitoring of a patient.

\section{OPEN ISSUES AND CuRrent Research Orientations}

\section{Sensor Network Architecture AND TOPOLOGY CONTROL}

Research Challenges Related to WSN Architecture One key aspect of WSN architecture and corresponding infrastructure is their complete dependence on application needs. One goal of the architecture is to allow components developed for one particular system to be used in other systems. The development of energy-efficient architectural solutions and their scalability are main open issues in the field of WSNs.

Wireless Hybrid Networks - Research on WSNs has employed architectures suiting theoretical and research needs: homogeneous, isolated, very large, multihop, and so on. In practice, wireless sensing and control need to be connected to the existing infrastructure through wireless body area networks (WBANs), wireless personal area networks (WPANs), WLANs, and beyond third generation (B3G) cellular or directly wired IP networks.

Current research has concentrated on the convergent integration of autonomous wireless networks (WLANs and WPANs), including multihop deployments with the infrastructure. Research was carried out on hierarchical WSNs with different classes of nodes [5]. However, very little has been done on the integration of WSNs, which often use proprietary wireless technologies and protocols, with other wireless networks. Thus, a challenging area is the study of wireless hybrid networks (WHNs), which represent the merging of WSNs and cellular systems (Fig. 4).

Management Aspects of WSNs - The design of efficient management software architecture for WSNs plays an important role. To decouple the development of applications from the hardware features of sensor devices, it is necessary to design a middleware layer that can perform vari- ous tasks. An efficient design, in order to optimize energy consumption and latency is clearly an outstanding challenge. Additional management aspects are discussed later.

Fundamental Issues and Trade-Offs in WSNs - Sensor networks should be deployed and operated in such a way that the sensor devices meet the following objectives:

- Must cope with bandwidth limitations

- Must use their limited energy judiciously in order to extend the network's lifetime

- Must monitor/measure and represent a physical process subject to specified accuracy

- The network must be fault-tolerant and allow a number of sensor node failures

The first goal is to identify analogies between source and channel coding, and some problems that arise in the context of WSNs. Then such similarities need to be exploited in both flat sensor network architectures and hierarchical WSN architectures.

Research Challenges Related to WSN Topology Control Topology control has several aspects: topology discovery, formation, and maintenance. Network topology is an important model of the network state as it implicitly gives information about the active nodes at a particular time as well as the connectivity/reachability map of the system.

Topological changes occur when a node disconnects/connects from/to all or some of its neighbors. Modification of the network structure may lead to performance degradations in the network. For this reason, network management procedures are required [7]. The management scheme has to be designed to keep the network infrastructure stable. Moreover, topology control applications can be closely related (e.g., clustering and routing or management and routing).

Topology Discovery and Network Formation - The main goal of topology discovery [8] is to determine sensing gaps and network coverage. It can also be used as a routing framework for calculating WSN characteristics: coverage, connectivity, usage patterns, and so on. Topology is also a key attribute since it aids in network performance analysis. Topology discovery algorithms have to retrieve network state information necessary for network administrators to monitor topology control decisions. One potential challenge is to optimize the mechanism for network formation in IEEE 802.15.4-based networks with tree topologies considering multisink scenarios.

Topology-Aware Routing - Routing protocols need to address many topological changes and high scalability. The aim is to study and propose routing mechanisms that are "aware" of the network topology. Special attention is paid to routing protocols that can be designed for the 802.15.4 standard. It is foreseen that we will investigate protocols for creation and maintenance of multicast topologies supporting data dissemination from multiple sources toward multiple observers. Node mobility is taken into account, too.

Clustering for Topology Control - A cluster tree provides a hierarchical routing structure in which messages are forwarded up along the tree from 
the source to the first common parent of both the source and destination nodes, and then down the tree to the destination. Efficient addressing of the nodes in a cluster tree can highly simplify the subjacent routing procedure. The addressing scheme and how the nodes of each branch are structured according to their particular range are in fact included in the ZigBee specification. The routing scheme needs improvement, especially in those cases where nodes in the vicinity of each other are organized in two branches of the tree and thus, instead of a direct link, a multihop path is required to communicate with both of them.

\section{Sensor Networks Protocols AND Data AgGREGATION}

Research Challenges Related to WSN Protocols - To design new protocols, the network needs to be analyzed under the characteristics of the subjacent architectures and applications that are used over WSNs. Additionally, the required trade-offs are quite different from those of traditional wireless networks. One of the most relevant aspects is power conservation; therefore, the required procedures should make the most efficient use of node energy. Another aspect is the usual low capacity of the devices, which poses some bounds on the complexity of the processes that can be employed. Last, but not least, appropriate sharing of the information throughout the whole protocol stack becomes a key aspect in order to tailor the operation of the protocol entities to the characteristics of the network.

In the following, the network protocol aspects on which the CRUISE project is focused are presented (Fig. 5).

Medium Access Control - The performance parameters used over traditional wireless networks are no longer valid for WSNs, in which energy consumption as well as behavior over spontaneous multihop topologies need to be considered. Medium access control (MAC) for WSNs is now dominated by the IEEE 802.15.4 protocol, for which the first commercial products exist. In this sense, the CRUISE project aims to analyze its shortcomings and propose new and advanced MAC techniques not currently covered.

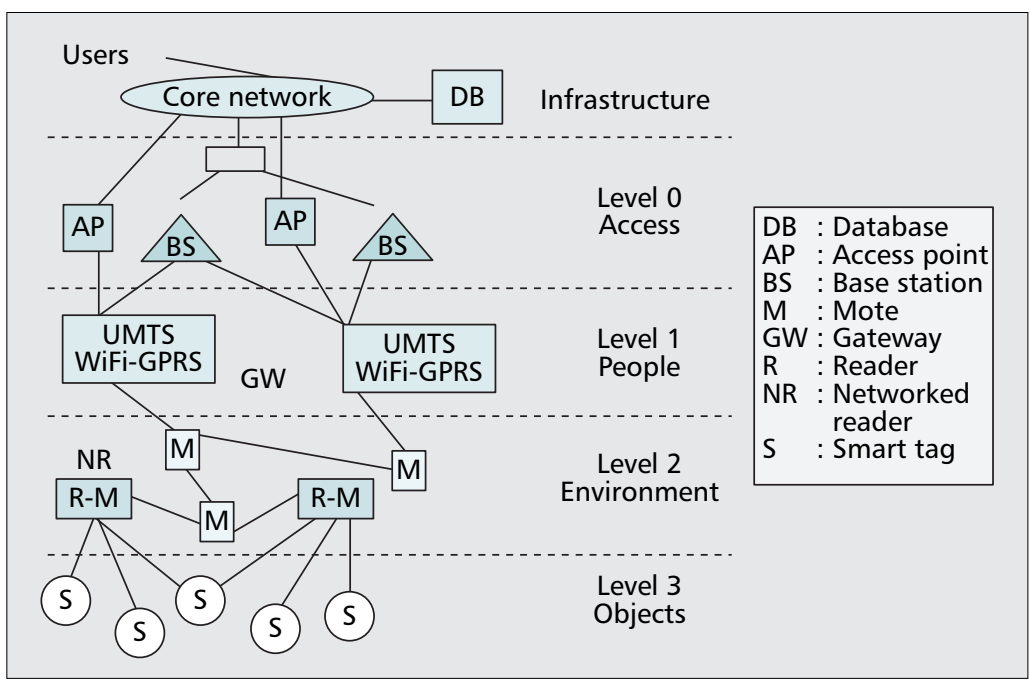

Figure 4. Wireless hybrid networks [6].

Routing Protocols - In this field there is no dominant solution yet. A lot of research has been dedicated to analysis of the behavior of protocols designed for ad hoc networks over WSNs, but the slight yet fundamental differences between these types of networks must be considered. Routing has to be adapted to the subjacent topology/architecture, and must consider the way in which sensor devices generate traffic, which is often either broadcast or multicast. On the other hand, typical performance metrics (e.g., throughput) are less important in the case of WSNs. Both reactive and proactive routing strategies and their impact on energy consumption are analyzed. Special attention is paid to the ZigBee architecture, since it is becoming the de facto standard for WSNs.

The CRUISE project structures its work on routing protocols on three main axes:

- Analyze routing strategies that provide optimized energy consumption.

- Study the impact clustering techniques may have on routing procedures.

- Investigate advanced routing techniques, embedding data querying information to optimize the required overhead.

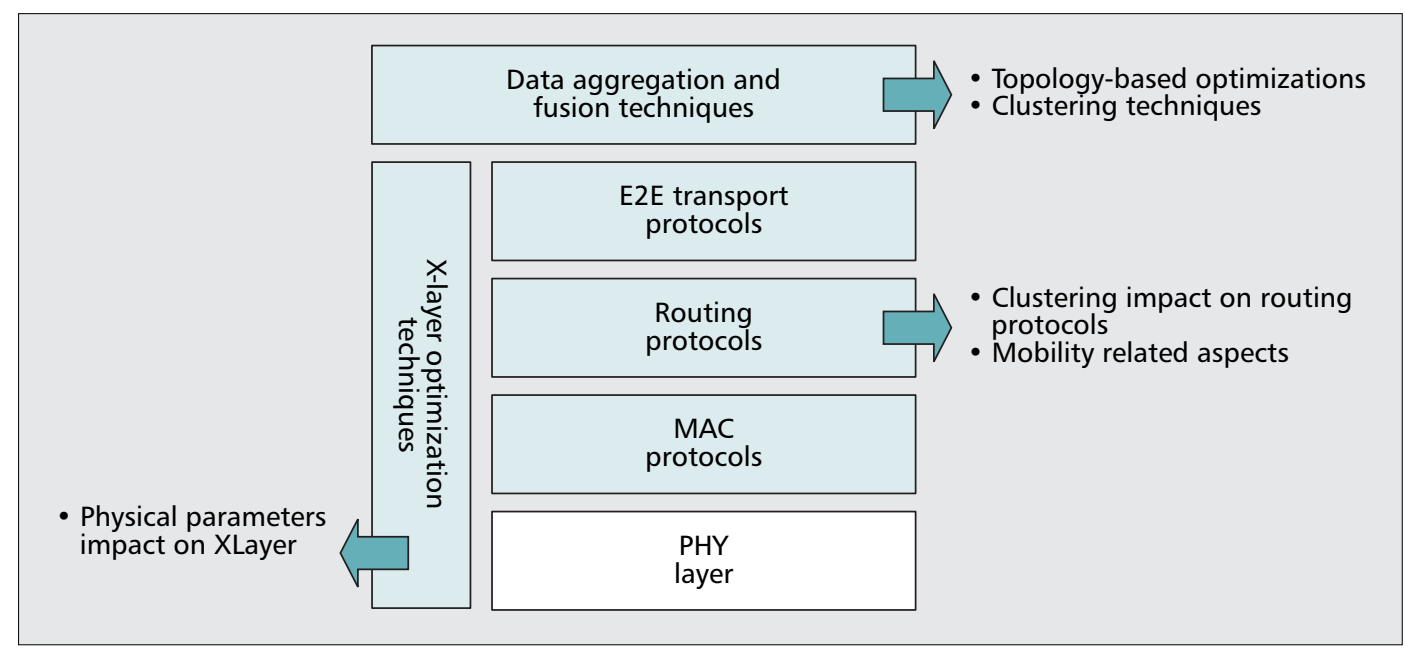

Figure 5. Research topics related to WSN protocols and data aggregation. 
End-to-End Transport Protocols - End-to-end transport protocols in WSNs are typically understood as mechanisms for reliable delivery of data from the source to the destination. This includes both recoveries from transmission errors and flow control mechanisms. Numerous techniques have been suggested, ranging from acknowledgmentbased mechanisms to forward error correction or repetition coding, and even multipath routing.

Several of the fundamental research issues for end-to-end transport in WSNs are still open. The flow control techniques studied so far are rather rudimentary and do not take advantage of available information (e.g., application-specific or topology information). Even less clear is how to best accomplish end-to-end transport in different types of many-to-one and many-to-many communications.

The CRUISE project investigates in depth how an adequate design of transport protocols may help to improve energy consumption and empirically assesses the compatibility problems with legacy TCP/IP protocols.

Cross-Layer Optimization in WSNs - Resource limitations typically found in WSN devices accentuate the need for algorithm optimizations in sensor network applications, most often to conserve energy. One technique for such optimizations is cross-layer interactions where, in contrast to a strictly layered architecture, different entities closely interact, dealing with the properties of sensor networks (e.g., impairments of radio propagation, limited battery lifetime) that cannot be appropriately handled by legacy architectures. Therefore, in WSNs the traditional layered approach is substituted by component-based architectures where cross-layer interactions are more easily performed.

However, the requirements for system support are still unclear. In fact, there is no common understanding of cross-layer interactions yet. In particular, two different approaches are followed:

- Integration of physical and MAC schemes (e.g., UWB and IEEE 802.15.4)

- Network layer optimizations, in which routing is based on geometrical optics (i.e., taking information from the physical layer)

Research Challenges Related to Data Aggregation and Fusion in WSNs - Traffic generated by WSN applications is typically characterized by a high degree of spatial correlation, because all the devices located within the area where a phenomenon occurs will notify the sink of the same event. To reduce its impact, data fusion techniques are proposed: combine the same phenomenon data coming from multiple sensors and turn them into a single packet. This allows reduction of energy consumption and thus an increase of the network's lifetime. The strategies for data fusion are centralized, tree-based, and clusterbased (static and dynamic).

The success of the aggregation procedure depends on different factors and protocol entities, and a single-layer strategy would produce only suboptimal solutions. Therefore, a crosslayer approach is more appropriate. Within CRUISE, the focus is on both tree-based and cluster-based schemes, discussing metrics such as overhead and the reliability of the subjacent aggregation schemes.

\section{SECURITY AND MOBILITY FOR WIRELESS SeNSOR NetWORKS}

Research Challenges Related to Security and Privacy Apart from the general security requirements for WSNs, some of the above mentioned scenarios concern collection and communication of very sensitive data for the individual. Thus, to properly protect network operation and not hinder acceptance of this promising technology, WSNs require novel security algorithms to achieve robustness and privacy while maintaining a good performance level. Three security aspects are the focus of the joint research: providing privacy and anonymity, robustness through misbehavior detection, and usability through authentication protocols for mobile nodes.

Privacy Protection and Secure Profile Management - In ubiquitous sensorized environments diverse types of sensitive data for individuals, such as their health, behavior, and location, will be generated and processed, so privacy and trust are key issues to address in the design of personcentric WSNs. In the medical scenario, for example, the patient's health status and medical history are communicated. In any case, people should be able to use services while preserving their privacy and anonymity. The diverse situations require different types of personal information to be disclosed, and context-aware policies with user-friendly mechanisms to specify different sets of rules for people's roles and identification of appropriate levels of protection for sensitive data. In this way the demand for the person to be in control of sensitive information disclosure can be fulfilled.

The direction taken by the partners is to develop an adaptive privacy protection concept with a profile management framework for WSNs that intelligently takes into account context, user roles, and preferences [9].

Detection of Misbehavior in WSNs and Attack Modeling Reliable and timely detection of deviation from legitimate protocol operation is recognized as a prerequisite for ensuring efficient use of resources and minimizing performance losses in WSNs. An attacker may exploit protocol weaknesses to obtain network resources to his/her own benefit by depriving others or simply to cause trouble to the network. Such attacks can be carried at any network layer, for example, by not respecting the MAC access rules, routing strategy, or transport layer congestion control algorithm.

The basic feature of attack and misbehavior strategies is that they are entirely unpredictable [10]. In the presence of such uncertainty and to evaluate security threats, it is meaningful to seek models and decision rules that are energy-efficient and robust under a wide range of uncertainty conditions, protocols, and information available.

It is also important to investigate ways of:

- Modeling the attack itself in a hierarchical manner, as proposed by attack trees 
- Modeling and quantifying risk and benefit for the attacker in order to identify the most probable attacks for each WSN application space

Secure Mobility: Authentication and Key Management Changing topology implies that the individual node needs to maintain information of the nodes entering and leaving its neighborhood, and minimize the number of re-authentications. Many security protocols and solutions for key management have been proposed, some of which are quite widely used. However, the cryptographic algorithms and security protocols used in WSNs must consider the storage and computational limitations of the nodes. Key management is also affected by the topology and internal organization of WSNs, which can be hierarchical, distributed, or hybrid. The intrinsic constraint of WSNs is that the number of messages used in authentication must not be excessive. Having this requirement in mind, centralized and distributed strategies and predistribution techniques are evaluated in the project.

Research Challenges Related to Mobility - Mobility introduces great demands on WSNs, but can also significantly increase the capability of WSNs by making them resilient to failures, reactive to events, and able to support disparate missions with a common set of sensors. The research issues for mobility in CRUISE are mobility models for predefined scenarios, and MAC and routing optimization under the different mobility classes and models.

Mobility Models for Predefined Scenarios - We investigate how different mobility models can be applied to simulate realistic scenarios for WSNs. A specific issue is to find algorithms that help various aspects of WSNs and provide support for some of the objectives for sensor mobility:

- Dynamic coverage: Mobile sensors can be used to cover undersampled areas.

- Event detection and tracking: Mobility may be useful to detect and track spatially and temporally spread events.

- Network repair: A faulty node could be replaced by moving a healthy one in its place.

- Data mules: This means having mobile nodes collecting data from stationary nodes.

- Distributed calibration: This can be achieved by calibrating stationary sensors.

MAC and Routing Optimization Under Different Mobility Classes and Models - Most of the proposed MAC protocols to date are not efficient for mobile WSNs with moderate to high mobility. The difficulty stems from the fact that the MAC protocol must be able to assign different priorities taking into account traffic information, the mobility pattern of the nodes, and their impact on higherlayer procedures such as routing. We study the effects of mobility, considering the realistic constraints (energy, CPU, and memory) and how the standards, especially IEEE 802.15.4, can incorporate a clever mobility-aware scheme. It is essential to investigate:

- Algorithms to predict potential topology changes based on mobility models

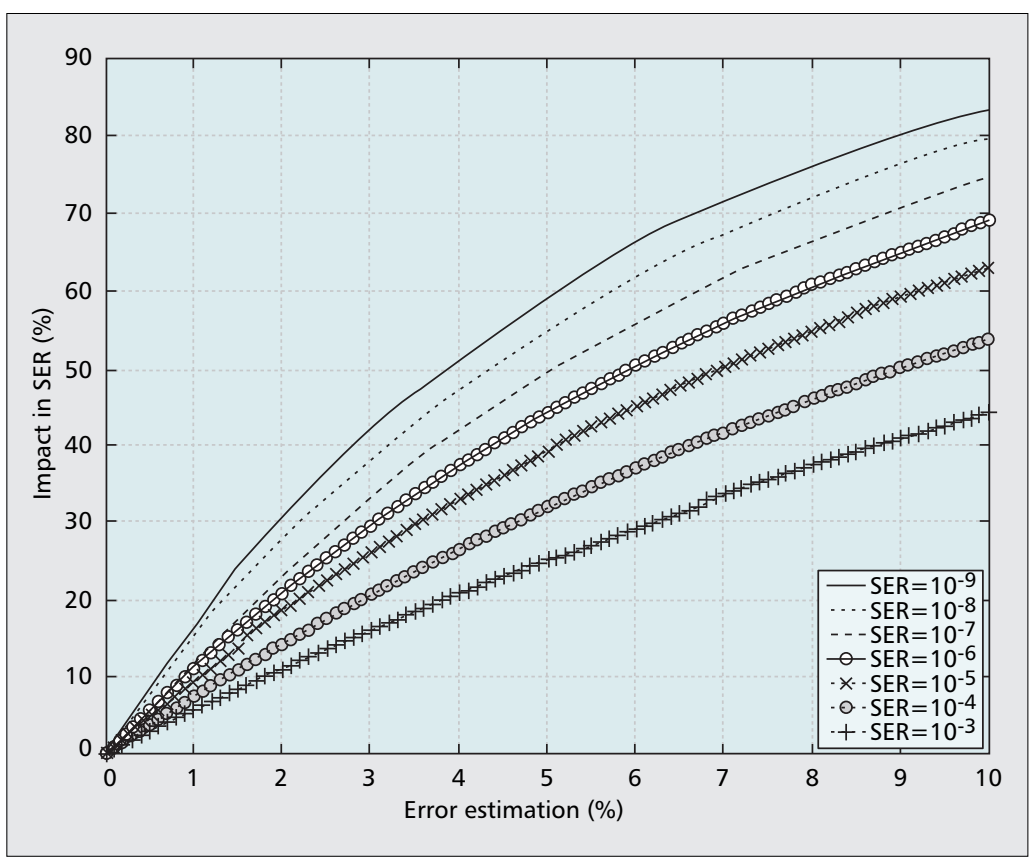

Figure 6. Impact of channel estimation errors on multi-user adaptation.

- A clever mobility-aware scheme in IEEE 802.15.4 for contention channel access/GTS slot assignment

- Routing protocols in hybrid sensor networks with stationary and mobile nodes

\section{Wireless Sensor NetWork TRANSMISSION ASPECTS}

Research Challenges Related to WSN Transmission - As pointed out in [2], the physical layer has been until now a relatively unexplored area in WSNs. Although a significant amount of research has been done on signal design and transmission techniques for wireless communication systems, including ad hoc networks, the findings and guidelines are not always the most appropriate to meet the unique features and application requirements of sensor networks.

In the following subsections we describe the related research issues within CRUISE concerning transmission and reception, which contribute to the deployment and success of WSNs.

Modulation and Coding for WSNs - The target within CRUISE is to develop modulation and coding schemes suitable for WSNs, and adequate to achieve the performance requirements and power constraints of WSNs, in both the singlelink case and a multi-user environment, with a focus on ultra-wideband (UWB) and multipleinput multiple-output (MIMO) techniques, both independently and combined.

UWB or impulse radio (IR) modulation has recently drawn considerable interest for shortrange communication applications. We aim to design:

- Higher-order modulation schemes for IRUWB signaling

- Efficient hopping strategies

- Error correction schemes and trellis coded modulation for UWB systems 
Techniques employing multiple antennas at the transmitter and/or receiver (MIMO) play a powerful role in improving the performance of and reducing the required transmission power for wireless systems. MIMO techniques applied to WSNs would significantly reduce the impact fading and can lead to significant power savings by exploiting diversity. The drawbacks: MIMO techniques are often highly complex and powerhungry. Thus, we examine MIMO techniques to determine if there is overall energy saving for the envisaged applications.

Finally, we look at the combination of UWB and MIMO since it will potentially provide both the low-energy behavior of the first and the spectral efficiency of the second.

Channel Estimation for WSNs - All the above mentioned techniques rely on a good channel estimation to perform coherent detection at the receiver. Channel estimates are necessary for physical layer processing, but are also of paramount importance for the other layers (MAC, network) in the framework of a crosslayer design. Therefore, the identification and design of adequate channel estimation schemes is of the utmost importance for WSNs. Moreover, channel estimation is mandatory, and even more accuracy is needed if we wish to adapt the transmitter to the channel conditions. Figure 6 shows the impact on the symbol error rate (SER) when a multi-user adaptive algorithm is performed in the presence of channel estimation errors. Small errors in the estimation procedure (e.g., 5 percent) can lead to 50 percent SER degradation or higher.

For these reasons we follow two approaches:

- Design channel adaptation algorithms that are resilient to estimation errors.

- Design and evaluate robust techniques for channel estimation in a distributed manner.

Blind vs. pilot-based schemes are considered, and estimation methods and pilot assignment that exploit the spatial correlation in channels for dense WSNs are explored.

Localization, Radio Frequency, and Propagation Issues Synchronization is a requirement in WSNs not only from the perspective of applications, but also to achieve diversity through cooperation among nodes. To provide algorithms for time delay estimation and localization dedicated to WSNs, electromagnetic field source recognition evaluation by correlation means is being explored, as well as the influence of the frequency and tissue properties on the communication path loss between sensors.

Further work is devoted to the development of the appropriate channel models to characterize the WSN environment. Most transmission research activities will find support on these models.

Collaborative Transmission - We focus on exploiting coordinated or collaborative transmission in WSNs, identifying the key parameters and analyzing the performance benefits of using such techniques in terms of: bit-rate, bandwidth, error probability and energy-efficiency. New distributed beamforming methods to provide can- cellation of interference and noise, assuming a randomly deployed distributed WSNs, are under design, paying particular attention to the feasibility of collaborative transmission solutions for IR-UWB communication due to the appropriateness of this technique for short-range communications.

Decentralized Source and Channel Coding - We work with decentralized source coding, using the correlation models based on the physical laws that determine the behavior of the environment measured by the WSNs, as well as self-organized and power-efficient methods for learning nonstationary correlation models. Joint source-channel codes are also developed, motivated by the communication paradigm, aiming at higher efficiency in power, delay, and complexity.

\section{CONCLUSIONS AND FUTURE WORK}

The primary objective of CRUISE is to make a significant contribution to the effectiveness of research on communication and application aspects of WSNs in Europe. This article describes the research strategy and roadmap within the context of CRUISE objectives. The project integration work is grouped in four main directions: architectures and topology control, protocols and data fusion, mobility and security, and transmission. From the broad spectrum of open topics within each direction, the partners have identified key research issues for joint work. Work in progress in each area is presented in the context of the state of the art, and directions of future efforts are suggested.

Follow-up topics spreading over several investigated areas, key for the common project framework based on the integration of WSNs with wireless hybrid networks, are on top of the research agenda for the evolution of research.

\section{ACKNOWLEDGMENTS}

This work has been performed in the framework of the IST-4-027738 NoE CRUISE, which is partly funded by the European Commission. The authors acknowledge the contribution of their colleagues from the consortium.

\section{REFERENCES}

[1] T. Arampatzis and J. Lygeros, "A Survey of Applications of Wireless Sensors and Wireless Sensor Networks," Proc. 13th Med. Conf. Control and Automation, Limassol, Cyprus, June 27-29, 2005, pp. 719-24.

[2] I. Akyildiz et al., "A Survey on Sensor Networks," IEEE Commun. Mag., Aug. 2002, pp. 102-14.

[3] M. P. Michaelides and C. G. Panayiotou, "Event Detection Using Sensor Networks," 45th IEEE Conf. Decision and Control, Dec. 2006, pp. 6784-89.

[4] A. Timm-Giel et al., "Wireless and Ad Hoc Communications Supporting the Firefighter," Proc. 15th IST Mobile and Wireless Summit, Mykonos, Greece, 4-8 June 2006.

[5] R. Chandra, C. Fetzer, and K. Hogstedt, "Adaptive Topology Discovery in Hybrid Wireless Networks," Proc. Informatics, 1 st Int'l. Conf. Ad Hoc Networks and Wireless, Toronto, Canada, vol. 16, Sept. 20-22, 2002, pp 1-16.

[6] R. Verdone, V. Corvino, and J. Orriss, "A Hierarchical Hybrid Network Model," Proc. IEE 3G\&Beyond, London, U.K., Nov. 7-9, 2005.

[7] F. J. Claudios et al., "Performance Study of Reconfiguration Algorithms in Cluster-Tree Topologies for Wireless Sensor Networks," 12th Int'l. Wksp. Comp. Aided Modeling and Design of Commun. Links and Networks, Athens, Greece, 7 Sept., 2007; part of PIMRC 2007. 
[8] B. Deb, S. Bhatnagar, and B. Nath, "A Topology Discovery Algorithm for Sensor Networks with Applications to Network Management," Proc. IEEE CAS Wksp. Wireless Commun. and Networking, Pasadena, CA, Sept. 2002.

[9] A. Mitseva et al., "Context-Aware Adaptive Privacy Protection for Wireless Sensor Networks," Proc. 9th Int'l. Symp. Wireless Personal Multimedia Commun., San Diego, CA, Sept. 2006, pp. 1032-36.

[10] S. Radosavac, J. S. Baras, and I. Koutsopoulos, "A Framework for MAC Layer Misbehavior Detection in Wireless Networks," Proc. 4th ACM Wksp. Wireless Sec., Cologne, Germany, Sept. 02, 2005, pp. 33-42.

\section{BIOGRAPHIES}

ANELIA Mitseva (mitseva@es.aau.dk) received her Master's Degree in intelligent multimedia from Aalborg University in 2001. She works in the wireless security and sensor networks group, CTIF, Aalborg University, Denmark, and is technical manager of FP6 NoE CRUISE. She has been heavily involved in a number of projects funded by the European Commission, performing management and research tasks. Recent research interests are wireless sensor networks, security, privacy, usability and user friendliness.

Petia Todorova (Petia.Todorova@fokus.fraunhofer.de) received a Ph.D. degree from the Technical University-Sofia. Since 1988 she has been a senior scientist with Fraunhofer FOKUS, Berlin, Germany, participating in ITU-T efforts and a large number of international projects. She was appointed for 2002-2006 adjunct associate professor, Department of Computer Science, ODU, Norfolk, Virginia. Recent research interests include topology control in WSNs and resource allocation in satellite networks. She has published extensively in various conference proceedings and journals.

RAMON AGÜERO (ramon@tlmat.unican.es) received a degree in telecommunications engineering from the University of Cantabria in 2001 and a Ph.D. in 2008. He is an assistant lecturer in the Communications Engineering Department of that university. He has participated in several collaborative research projects. His research focuses on WLAN and WPAN technologies, with special attention on performance analysis of TCP/IP protocols over them, and multihop (mesh) networks. He is also interested in aspects concerning heterogeneous network deployments and the area of WSNs.

ANA GARCIA ARMADA (agarcia@tsc.uc3m.es) received a telecommunication eengineer degree from the Polytechnic University of Madrid, Spain, in July 1994 and a Ph.D. in electrical engineering from the same university in February 1998. She is currently working as an associate professor at the University Carlos III of Madrid, Spain, where she has occupied several management positions. Her research interests are multicarrier and MIMO techniques, and their application to several communication systems.

ChRISTOS PANAYIOTOU (christosp@ucy.ac.cy) received B.Sc. and Ph.D. degrees in electrical and computer engineering (ECE) from the University of Massachusetts at Amherst, in 1994 and 1999, respectively. Currently, he is an aassistant professor with the ECE Department at the University of Cyprus. His research interests include wireless, ad hoc, and sensor networks, computer communication networks, quality of service provisioning, and optimization and control of discrete-event systems. He is an Associate Editor for the Conference Editorial Board of the IEEE Control Systems Society.

ANDREAS TIMM-GIEL (atg@comnets.uni-bremen.de) (M.Sc. 1994, Ph.D. 1999) worked as a researcher and project leader in mobile satellite communications at Bremen University from 1994 to 1999. Afterward he was a project manager at MediaMobil GmbH. Since late 2002 he has been senior researcher and lecturer in communication networks at the University of Bremen. Here he is also directing the Concerted Activity Adaptive Communications of TZI. His research focuses on sensor networks, and wireless and mobile communication networks.

LEONARDO MACCARI (maccari@lart.det.unifi.it) received a degree from the Engineering School of Florence University with a degree in computer engineering in 2004 . He joined the Dipartimento di Elettronica e Telecomunicazioni in 2005 as a research fellow. His current research interests are security aspects of wireless telecommunications, with special focus on mesh, sensor, and P2P networks.

NeEl RASHMI PRASAD (np@es.aau.dk) is an associate profes sor and head of the wireless security and sensor networks group, CTIF, Aalborg University. She is project coordinator of FP6 NoE CRUISE and leader of the EC Cluster for MSNs. Her research interests are mobility management; network management, CRN, security, privacy, and trust. She has coedited and co-authored two books, WLAN Systems and Wireless IP for Next Generation Communications and Wireless LANs and Wireless IP Security, Mobility, QoS and Mobile Network Integration (Artech House, 2001 and 2005). 